\title{
$\beta$-Carotene, A Potent provitamin A Carotenoids in the Amelioration of the Effects of Heroin
}

\section{Prem Kishore Saha, Umesh C. Goswami*and Swapan Kumar Dutta}

Department of Zoology, Retinoids Research Programme, DST-FIST (Govt. of India) \& UGC- SAP Sponsored, Gauhati University, Gauhati-781014, Assam, India

\begin{abstract}
$\beta$-Carotene, a potent provitamin A carotenoid having multifaceted physiological activities, could ameliorate the different types of abnormal behaviour in rats after the administration of heroin. The administration of different doses of heroin, viz $\mathrm{LD}_{25}, \mathrm{LD}_{50}$ showed various degrees of abnormal conditions and behaviours such as depletion of body weight, retinol reserve, decrease of water intake, piloerection, drowsiness, wet shakes, restlessness and jumping etc. $\beta$-carotene accounts for the amelioration of the above conditions and abnormal behaviour to a certain extent along with restoration of the retinol level in the liver of albino rats receiving heroin of different doses.
\end{abstract}

Keywords: Narcotic drug; Carotenoids; Retinoids; Drug abuse; Vitamin A

\section{Introduction}

The manifold effects of narcotic drugs on various metabolic processes have been known for a long time. Heroin is a narcotic drug which causes serious problems in the human health as well as those pertaining to a series of social problems. Weight loss, reduced food, water intake, vitamin deficiencies, anaemia, general malnutrition, gastro- intestinal disorders as well as effect [1-3]. Heroin addicted rats showed many withdrawal syndromes that can be divided into two phases as- (i) early or primary abstinence and (ii) protracted abstinence [4]. Besides that, heroin affects writhing apoptosis, piloerection, continuation of wet shake and aggression [5-7]. During the protracted phase, food, water intake, body weight, temperature and locomotion activities increase and persist for a long period [8,9]. $\beta$-Carotene, a provitamin A carotenoids, potent antioxidant, immunomodulator and growth promoter, has manifold properties to ameliorate the effects of various harmful agents, xenobiotics has been tested. We report here the $\beta$-carotene can ameliorate several effects of heroin after feeding both $\mathrm{LD}_{25}$ and $\mathrm{LD}_{50}$ doses.

In the present study an attempt has been made to study the effects of $\mathrm{LD}_{25}$ and $\mathrm{LD}_{50}$ doses of heroin on the growth and retinol reserve in liver of albino rats along with the amelioration of the effects of heroin in regulation of growth, water intake, piloerection, drowziness, itching, jumping, wet shakes and weight loss.

\section{Materials and Method}

\section{Maintenance of rats}

Young male albino rats (Rattus albino, Sprague dawley) of weight about $100-110 \mathrm{~g}$ were taken and were maintained at $27-30^{\circ} \mathrm{C}$ in plastic cages (18 cm X $12 \mathrm{~cm} \mathrm{X} 6 \mathrm{~cm}, 10$ animals/cage) in the animal house of the Zoology Department, Gauhati University, India. The rats were maintained on the diets ( $10 \mathrm{~g} / 100 \mathrm{~g}$ body weight $)$ as described earlier [10] taking the reference diet referred by [11] and termed as control diet. $0.5 \mathrm{ml}$ of ground nut oil containing $50 \mu \mathrm{g} \beta$-carotene was fed, $(0.0001863 \mathrm{M})$ to each rat daily studying its effects in combating the effects of various doses of heroin.

The rats were properly maintained with different feeds, cleaned every day, provided clean filtered water supplied in graduated plastic bottles. The rats maintained in these conditions [10] showed normal and satisfactory growth without any abnormalities .The rats were acclimatized to the control and $\beta$-carotene added diets for 2 weeks and the effects of $\mathrm{LD}_{25}$ and $\mathrm{LD}_{50}$ doses of heroin were tested in rats maintained in the above mentioned diets containing either $\beta$-carotene or in absence of $\beta$-carotene for another 7 weeks. The effects of different diets and heroin administration were repeated in triplicate and its metabolism on the regulation of growth and retinol reserves were recorded.

\section{Administration of heroin}

$\mathrm{LD}_{25}$ and $\mathrm{LD}_{50}$ dose of heroin was calculated following the method developed by us $[10,12]$ and found to be $0.0833 \mathrm{mg}$ and $0.167 \mathrm{mg} / 100 \mathrm{~g}$ body weight respectively.

In order to study the effects of heroin on various behaviours and its possible amelioration with $\beta$-carotene, the intramuscular injection of heroin were administered in the morning $(7 \mathrm{am})$ and various behaviours were observed. Different withdrawal symptoms were measured from the earlier works as shown piloerection [13], itching, drowsing, wet shakes [14], and jumping [15]. The intensity of all symptoms was measured through various degrees of behaviour showing doubtful (-), low $(+)$, moderate $(++)$ to high $(+++)$ or highest $(++++)$ along with the $\%$ of animals affected or showed abnormal behaviours from 0 to 6 hours.

\section{Chemicals}

Crystalline $\beta$-carotene, retinol, Retinyl acetate, and retinoic acid were obtained from F. Hoffman La- Roche, Basel, Switzerland, BASF - Germany. Other chemicals of analytical grade were purchased from British Drug House (B.D.H.), Laboratory Chemicals Division, Glaxo Laboratories (India) Ltd., Bombay and from Sigma-Aldrich India, Heroin was obtained from Assam Forensic Science Laboratory.

\section{Estimation of vitamin-A (Retinol) in rat Liver}

The animals were sacrificed under anesthesia and the livers were dissected. Extraction of retinol was done in diffused light following the method of [16]. Liver tissue (5 g) was grounded with five times anhydrous $\mathrm{Na}_{2} \mathrm{SO}_{4}$ and made into a dry powder. All retinoids and

*Corresponding author: U.C.Goswami, Principal Investigator of the Retinoids Research Scheme, Guwahati University, Guwahati, Assam, India, E-mail: ucgoswami@rediffmail.com

Received May 27, 2012; Accepted June 27, 2012; Published July 02, 2012

Citation: Saha PK, Goswami UC, Dutta SK (2012) $\beta$-Carotene, A Potent provitamin A Carotenoids in the Amelioration of the Effects of Heroin. Vitam Trace Elem 1:107.

Copyright: ( 2012 Saha PK, et al. This is an open-access article distributed unde the terms of the Creative Commons Attribution License, which permits unrestricted use, distribution, and reproduction in any medium, provided the original author and source are credited. 
carotenoids were then extracted with light petroleum ether $\left(40^{\circ} \mathrm{C}-60^{\circ}\right.$ C) in darkness and evaporated it to dryness. Alkaline hydrolysis was made by alcoholic $\mathrm{KOH}$ solution by keeping it overnight in darkness at low temperature. It was then extracted with diethyl ether and dried over anhydrous $\mathrm{Na}_{2} \mathrm{SO}_{4}$ Diethyl ether was evaporated and retinoids were stored in methanol at low temperature (below $-10^{\circ} \mathrm{C}$ ).

\section{HPLC}

The HPLC were conducted using reversed phase system. (Column- $\mu$ Bondapak $\mathrm{C}_{18}$,Water, $300 \mathrm{~mm}$ x $4.6 \mathrm{~mm}, 5 \mu$; mobile phase: Acetonitrile $(50 \mathrm{mM})$ : Ammonium acetate ( $\mathrm{pH}$ 6.9), Detector: 135-diode array detector (Perkin Elmer); Integrator: LCI 100 (Perkin Elmer); Flow rate of the eluent: $1.2 \mathrm{ml} / \mathrm{min}$.

The HPLC system consists of Perkin Elmer 250 binary pump. The Perkin Elmer 135-diode array detector was set at $315 \mathrm{~nm}$. The instrument was fitted with Rhyodine injector with a loop of $20 \mu \mathrm{l}$. The lowest limit of detection for each assay was $0.2 \mathrm{pmol}$. The amount of vitamin-A (retinol) present in the liver was estimated.

\section{Results}

The effects of the different diets containing $\beta$-carotene with heroin of different doses and in absence of $\beta$-carotene were compared with the animals reared with normal or control diets from its initial start, viz 0 day to 7 weeks. $\beta$-carotene showed better growth along with high retinol reserve in the livers of rats treated with the $\beta$-carotene added diets. $\beta$-carotene helped in maintaining a normal retinol reserve and growth to some extent in rats administered with $\mathrm{LD}_{25}$ dose and reserve and poor growth in $\mathrm{LD}_{50}$ doses of heroin respectively. In the absence of $\beta$-carotene, heroin treated groups of both $\mathrm{LD}_{25}$ and $\mathrm{LD}_{50}$ dose showed poor growth and loss of retinol from their livers (Table 1). Weekly examination of body weight and retinol reserves was conducted. Retinol reserve was estimated from its HPLC profiles as described earlier. It may be mentioned that $\beta$-carotene or $\beta$-carotene added diet did not show any toxic effects on the experimental rats.

Several behavioural changes such as water intake, piloerection, itching, drowsiness, jumping and wet shake etc. were recorded after the administration of heroin of different doses. It may be mentioned that control diets, ground nut oil and $\beta$-carotene added diets did not show any abnormal behaviours that has been monitored from 0-6 hours. However the abnormal behaviours were encountered in rats administered with different doses of heroin added with $\beta$-carotene, or in absence of $\beta$-carotene. It has been found that $\beta$-carotene can ameliorate the above mentioned drug-induced behaviours. No abnormal behaviours have been recorded after 6 hours. The details are shown in (Table 2-6).

\section{Discussion}

Drug-nutrient interactions is a significant issue, where such interactions may interfere with the pharmacokinetics process and the nutrition status plays an important role in a drug's pharmacologic response which may interfere with the pharmacokinetic process [1719]. Increasing attention has been focused on nutrient-drug interaction because many drugs have become more potent and have greater specificity and with extended duration have increased the incidence of nutrient-drug interactions. Drug abuse has become a great social menace where it involved a series of social, health and other economic situation of the involved persons as well as society. Further it has been seen that vitamin A deficiency is also a great problem in South East Asian countries. Considering all these collective issues and problems we have shown an interaction of heroin and $\beta$-carotene, where $\beta$ can ameliorate certain effects of heroin administration in animals taking rat as a model.

$\beta$-carotene supplementation has no apparent effects either in weight loss or depletion of retinol reserves as well as on expression of unusual behaviours. Increased dietary $\beta$-carotene or other provitamin $\mathrm{A}$ carotenoids may effects the steady-state concentration in body fluid or tissues and serve as localised substrates for retinoids formation. Retinol which exerts striking effects on diverse functions such as growth, development, and differentiation can be exerted from $\beta$-carotene. The present studies have been designed to determine whether an interaction exists between heroin and $\beta$-carotene or its metabolites to effect the growth and expression of regulatory activities in amelioration of unusual or abnormal activities that has been encountered after the administration of different doses of heroin.. The decrease of weight after administration of heroin $\left(\mathrm{LD}_{25}\right.$ and $\left.\mathrm{LD}_{50}\right)$ in the animal is found to be consistent with earlier findings $[9,14,20]$. Two possible mechanisms may act (i) altered level of anabolic hormones [21] or (ii) decrease of food intake [22]. Further, the increased physical activity or other undefined factors in heroin treated rats might of some importance in causing body weight loss. The support of $\beta$-carotene in the increase of body weight and in the prevention of loss of body weight by heroin

\begin{tabular}{|c|c|c|c|c|c|c|c|c|c|c|c|c|c|c|}
\hline \multirow[t]{2}{*}{ Week } & \multicolumn{2}{|c|}{ Control diet (CD) } & \multicolumn{2}{|c|}{ Control diet +Oil } & \multicolumn{2}{|c|}{$\begin{array}{l}\text { Control diet + } \\
\beta \text {-carotene }\end{array}$} & \multicolumn{2}{|c|}{$\begin{array}{c}\text { Control diet }+ \\
\beta \text {-carotene+ Heroin } \\
\left(\operatorname{LD}_{25}\right)\end{array}$} & \multicolumn{2}{|c|}{$\begin{array}{c}\text { Control diet }+\beta- \\
\text { carotene }+\mathrm{H}\left(\mathrm{LD}_{50}\right)\end{array}$} & \multicolumn{2}{|c|}{$\begin{array}{c}\text { Control diet +Heroin } \\
\left(\mathrm{LD}_{25}\right)\end{array}$} & \multicolumn{2}{|c|}{ Control diet + Heroin $\left(\operatorname{LD}_{50}\right)$} \\
\hline & Wt(g) & Retinol $(\mu \mathrm{g})$ & $\mathrm{Wt}(\mathrm{g})$ & Retinol $(\mu \mathrm{g})$ & $\mathrm{Wt}(\mathrm{g})$ & Retinol $(\mu \mathrm{g})$ & $W t(g)$ & Retinol $(\mu \mathrm{g})$ & $W t(g)$ & $\begin{array}{c}\text { Retinol } \\
(\mu \mathrm{g})\end{array}$ & Wt(g) & $\begin{array}{c}\text { Retinol } \\
(\mu \mathrm{g})\end{array}$ & $\mathrm{Wt}(\mathrm{g})$ & Retinol $(\mu \mathrm{g})$ \\
\hline 0 & $\begin{array}{l}110.5 \\
\pm 2.5\end{array}$ & $\begin{array}{l}23.2 \\
\pm 0.5\end{array}$ & $\begin{array}{r}114 \\
\pm 3.5\end{array}$ & $\begin{array}{l}23.5 \\
\pm 1.5\end{array}$ & $\begin{array}{r}113 \\
\pm 3.5\end{array}$ & $\begin{array}{c}23 \\
\pm 0.5\end{array}$ & $\begin{array}{r}109 \\
\pm 2.5\end{array}$ & $\begin{array}{l}23.5 \\
\pm 0.5\end{array}$ & $\begin{array}{r}112 \\
\pm 3.5\end{array}$ & $\begin{array}{c}24 \\
\pm 0.5\end{array}$ & $\begin{array}{c}114 \\
\pm 2.0\end{array}$ & $\begin{array}{l}23.5 \\
\pm 0.5\end{array}$ & $\begin{array}{r}113 \\
\pm 3.2\end{array}$ & $\begin{array}{l}24.2 \\
\pm 1.0\end{array}$ \\
\hline 2 & $\begin{array}{l}122^{*} \\
\pm 2.5\end{array}$ & $\begin{array}{l}29^{*} \\
\pm 1.0\end{array}$ & $\begin{array}{l}125^{*} \\
\pm 3.5\end{array}$ & $\begin{array}{l}28.5^{*} \\
\pm 0.5\end{array}$ & $\begin{array}{l}131^{*} \\
\pm 3.5\end{array}$ & $\begin{array}{l}117^{*} \\
\pm 4.0\end{array}$ & $\begin{array}{l}135^{*} \\
\pm 3.5\end{array}$ & $\begin{array}{l}119^{*} \\
\pm 5.5\end{array}$ & $\begin{array}{l}138^{*} \\
\pm 2.5\end{array}$ & $\begin{array}{l}116^{*} \\
\pm 7.0\end{array}$ & $\begin{array}{l}136^{*} \\
\pm 1.5\end{array}$ & $\begin{array}{c}30^{*} \\
\pm 1.0\end{array}$ & $\begin{array}{l}134^{*} \\
\pm 3.0\end{array}$ & $\begin{array}{c}30^{*} \\
\pm 1.5\end{array}$ \\
\hline 3 & $\begin{array}{r}130 \\
\pm 3.0\end{array}$ & $\begin{array}{l}31^{*} \\
\pm 1.0\end{array}$ & $\begin{array}{l}134^{*} \\
\pm 7.0\end{array}$ & $\begin{array}{c}32 \\
\pm 1.5\end{array}$ & $\begin{array}{l}143^{*} \\
\pm 4.5\end{array}$ & $\begin{array}{r}123 \\
\pm 5.0\end{array}$ & $\begin{array}{r}138 \\
\pm 7.0\end{array}$ & $\begin{array}{c}124 \\
\pm 6.5\end{array}$ & $\begin{array}{r}132 \\
\pm 5.5\end{array}$ & $\begin{array}{r}91^{*} \\
\pm 2.5\end{array}$ & $\begin{array}{l}126^{*} \\
\pm 3.5\end{array}$ & $\begin{array}{c}24^{*} \\
\pm 0.5\end{array}$ & $\begin{array}{l}124^{*} \\
\pm 5.5\end{array}$ & $\begin{array}{c}21^{*} \\
\pm 0.5\end{array}$ \\
\hline 4 & $\begin{array}{l}138^{*} \\
\pm 3.0\end{array}$ & $\begin{array}{c}34^{*} \\
\pm 1.5\end{array}$ & $\begin{array}{l}142^{*} \\
\pm 4.5\end{array}$ & $\begin{array}{c}35 \\
\pm 2.5\end{array}$ & $\begin{array}{l}158^{*} \\
\pm 4.5\end{array}$ & $\begin{array}{r}130 \\
\pm 7.5\end{array}$ & $\begin{array}{r}142 \\
\pm 5.5\end{array}$ & $\begin{array}{r}130 \\
\pm 3.5\end{array}$ & $\begin{array}{c}127 \\
\pm 5.5\end{array}$ & $\begin{array}{c}79^{*} \\
\pm 3.5\end{array}$ & $\begin{array}{l}118^{*} \\
\pm 5.5\end{array}$ & $\begin{array}{c}15^{*} \\
\pm 0.5\end{array}$ & $\begin{array}{l}119^{*} \\
\pm 3.5\end{array}$ & $\begin{array}{c}12^{*} \\
\pm 0.5\end{array}$ \\
\hline 5 & $\begin{array}{l}145^{*} \\
\pm 6.5\end{array}$ & $\begin{array}{c}37^{*} \\
\pm 0.5\end{array}$ & $\begin{array}{l}148^{*} \\
\pm 5.5\end{array}$ & $\begin{array}{c}36 \\
\pm 0.5\end{array}$ & $\begin{array}{l}136^{*} \\
\pm 7.5\end{array}$ & $\begin{array}{l}136^{*} \\
\pm 5.5\end{array}$ & $\begin{array}{l}146^{*} \\
\pm 3.5\end{array}$ & $\begin{array}{l}137^{*} \\
\pm 4.5\end{array}$ & $\begin{array}{c}124 \\
\pm 6.5\end{array}$ & $\begin{array}{c}74^{*} \\
\pm 0.5\end{array}$ & $\begin{array}{r}116 \\
\pm 5.5\end{array}$ & $\begin{array}{c}13^{*} \\
\pm 0.5\end{array}$ & $\begin{array}{l}115^{*} \\
\pm 5.5\end{array}$ & $\begin{array}{c}10^{*} \\
\pm 0.5\end{array}$ \\
\hline 6 & $\begin{array}{l}155^{*} \\
\pm 3.5\end{array}$ & $\begin{array}{c}38 \\
\pm 0.5\end{array}$ & $\begin{array}{l}155^{*} \\
\pm 5.5\end{array}$ & $\begin{array}{c}39^{*} \\
\pm 1.0\end{array}$ & $\begin{array}{l}170^{*} \\
\pm 5.5\end{array}$ & $\begin{array}{l}141^{*} \\
\pm 4.5\end{array}$ & $\begin{array}{l}150^{*} \\
\pm 3.5\end{array}$ & $\begin{array}{l}130^{*} \\
\pm 7.5\end{array}$ & $\begin{array}{l}121^{*} \\
\pm 4.5\end{array}$ & $\begin{array}{c}65^{*} \\
\pm 1.5\end{array}$ & $\begin{array}{r}113 \\
\pm 5.5\end{array}$ & $\begin{array}{c}10 \\
\pm 0.5\end{array}$ & $\begin{array}{l}112^{*} \\
\pm 2.5\end{array}$ & $\begin{array}{c}8 \\
\pm 0.5\end{array}$ \\
\hline 7 & $\begin{array}{l}160^{*} \\
\pm 4.5\end{array}$ & $\begin{array}{c}39^{*} \\
\pm 1.5\end{array}$ & $\begin{array}{l}163^{*} \\
\pm 1.5\end{array}$ & $\begin{array}{c}41^{*} \\
\pm 0.5\end{array}$ & $\begin{array}{l}176^{*} \\
\pm 2.5\end{array}$ & $\begin{array}{l}148^{*} \\
\pm 3.5\end{array}$ & $\begin{array}{l}156^{*} \\
\pm 1.5\end{array}$ & $\begin{array}{c}1128^{*} \\
\pm 0.5\end{array}$ & $\begin{array}{l}119^{*} \\
\pm 0.5\end{array}$ & $\begin{array}{c}60^{*} \\
\pm 1.0\end{array}$ & $\begin{array}{l}112^{*} \\
\pm 1.0\end{array}$ & $\begin{array}{l}8.25^{*} \\
\pm 0.5\end{array}$ & $\begin{array}{l}108^{*} \\
\pm 0.5\end{array}$ & $\begin{array}{l}6.25^{*} \\
\pm 0.5\end{array}$ \\
\hline
\end{tabular}

Table 1: Change of body weight and retinol reserve of control and treated animal of different diets. (10 rats in each cage, repeated in three sets of experiments for each diets) The results are the mean and \pm SD 3 sets of experiments of each diets; * significant at $P<0.05 \%$. 
Citation: Saha PK, Goswami UC, Dutta SK (2012) $\beta$-Carotene, A Potent provitamin A Carotenoids in the Amelioration of the Effects of Heroin. Vitam Trace Elem 1:107.

Page 3 of 5

\begin{tabular}{|c|c|c|c|c|c|c|c|}
\hline $\begin{array}{c}\text { Day of } \\
\text { administration }\end{array}$ & Control diet (CD) & Control diet +Oil & $\begin{array}{c}\text { Control diet }+ \\
\beta-c\end{array}$ & $\begin{array}{c}\text { Control diet }+\beta-\mathrm{c}+ \\
\quad \mathrm{H}\left(\mathrm{LD}_{25}\right)\end{array}$ & Control diet $+\beta-c+H\left(L_{50}\right)$ & Control diet $+\mathrm{H}_{25}\left(\mathrm{LD}_{25}\right)$ & $\begin{array}{l}\text { Control diet } \\
+\mathrm{H}_{50}\left(\mathrm{LD}_{50}\right)\end{array}$ \\
\hline 1. & $61.5 \pm 0.5$ & $61 \pm 1.0$ & $62.5 \pm 0.5$ & $62 \pm 0.5$ & $62 \pm 0.5$ & $61.5 \pm 0.5$ & $63 \pm 0.5$ \\
\hline 2 & $62 \pm 0.2$ & $62.5 \pm 0.4$ & $63 \pm 0.3$ & $63 \pm 0.5$ & $62.5 \pm 0.5$ & $62 \pm 0.5$ & $62.5 \pm 0.5$ \\
\hline 3 & $62 \pm 0.2$ & $62 \pm 0.5$ & $63 \pm 0.5$ & $63.5 \pm 0.6$ & $62 \pm 0.2$ & $63 \pm 0.5$ & $64 \pm 0.2$ \\
\hline 4 & $63 \pm 0.5$ & $62.5 \pm 0.5$ & $63 \pm 0.2$ & $64 \pm 0.3$ & $63 \pm 0.5$ & $64 \pm 0.5$ & $66.6 \pm 0.4$ \\
\hline 5 & $63.5 \pm 0.5$ & $62.5 \pm 0.2$ & $65 \pm 0.5$ & $64 \pm 0.2$ & $64 \pm 0.5$ & $63 \pm 0.4$ & $66.5 \pm 0.5$ \\
\hline 6 & $64 \pm 0.2$ & $62.5 \pm 0.4$ & $65 \pm 0.5$ & $65 \pm 0.6$ & $64 \pm 0.5$ & $63 \pm 0.5$ & $63.5 \pm 0.2$ \\
\hline 7 & $63.5 \pm 0.5$ & $63.2 \pm 0.2$ & $67 \pm 1.0$ & $65.5 \pm 0.5$ & $65 \pm 0.5$ & $62 \pm 0.2$ & $68 \pm 0.5$ \\
\hline 8 & $64 \pm 0.2$ & $64 \pm 0.4$ & $68 \pm 0.5$ & $66 \pm 0.5$ & $66.5 \pm 0.5$ & $60 \pm 0.2$ & $60 \pm 0.2$ \\
\hline 9 & $65 \pm 0.5$ & $65.5 \pm 1.5$ & $70 \pm 1.5$ & $67 \pm 1.0$ & $67.0 \pm 1.0$ & $58 \pm 0.5$ & $57 \pm 0.5$ \\
\hline 10 & $65 \pm 0.5$ & $66 \pm 1.0$ & $7.5 \pm 1.0$ & $69 \pm 1.0$ & $60.0 \pm 0.5$ & $57 \pm 0.5$ & $56 \pm 0.5$ \\
\hline 11 & $66 \pm 0.5$ & $66 \pm 0.5$ & $71 \pm 1.5$ & $65 \pm 1.0$ & $58 \pm 0.5$ & $52 \pm 0.2$ & $53.5 \pm 0.5$ \\
\hline 12 & $69 \pm 1.0$ & $68 \pm 1.0$ & $71 \pm 1.5$ & $68 \pm 1.5$ & $57.5 \pm 0.5$ & $52 \pm 0.5$ & $53 \pm 0.2$ \\
\hline 13 & $70 \pm 1.0$ & $69 \pm 1.0$ & $72 \pm 1.5$ & $65 \pm 1.5$ & $59 \pm 0.5$ & $54 \pm 0.2$ & $50 \pm 0.5$ \\
\hline 14 & $71 \pm 1.5$ & $72 \pm 1.5$ & $74 \pm 2.5$ & $60.3 \pm 2.5$ & $41 \pm 0.5$ & $52.5 \pm 0.5$ & $40 \pm 0.2$ \\
\hline
\end{tabular}

Table 2: Amount of water intake $(\mathrm{ml})$ measured in rats fed with different diets containing different doses of heroin with $\beta$-carotene or without $\beta$-carotene. (10 rats in each cage, repeated in three sets of experiments for each diets, $n=14$ observation).

\begin{tabular}{|c|c|c|c|c|c|c|c|c|c|c|c|c|c|c|c|c|c|c|c|}
\hline \multirow[t]{2}{*}{$\begin{array}{l}\text { Days of } \\
\text { Heroin } \\
\text { Injection }\end{array}$} & \multirow{2}{*}{$\begin{array}{c}0 \\
0-6 h\end{array}$} & \multirow{2}{*}{ 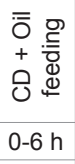 } & \multirow{2}{*}{ 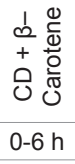 } & \multicolumn{4}{|c|}{$\begin{array}{c}\mathrm{CD}+\beta-\text { Carotene }+ \text { Heroin } \\
\left(\mathrm{LD}_{25}\right)\end{array}$} & \multicolumn{4}{|c|}{$\begin{array}{c}C D+\mid \beta-\text { Carotene + Heroin } \\
\left(\mathrm{LD}_{50}\right)\end{array}$} & \multicolumn{4}{|c|}{$C D+$ Heroin $\left(L_{25}\right)$} & \multicolumn{4}{|c|}{$C D+$ Heroin $\left(L_{50}\right)$} \\
\hline & & & & $0-1 \mathrm{~h}$ & $1-2 \mathrm{~h}$ & $3-4 \mathrm{~h}$ & $4-6 \mathrm{~h}$ & $0-1 \mathrm{~h}$ & $0-2 \mathrm{~h}$ & $3-4 \mathrm{~h}$ & $4-6 \mathrm{~h}$ & $0-1 \mathrm{~h}$ & $0-2 \mathrm{~h}$ & $3-4 \mathrm{~h}$ & $4-6 \mathrm{~h}$ & $0-1 \mathrm{~h}$ & $0-2 \mathrm{~h}$ & $3-4 \mathrm{~h}$ & $4-6 \mathrm{~h}$ \\
\hline $1^{\text {st }}$ & $\mathrm{N}$ & $\mathrm{N}$ & $\mathrm{N}$ & $\mathrm{N}$ & $\mathrm{N}$ & $\mathrm{N}$ & $N$ & $N$ & $\mathrm{~N}$ & $\mathrm{~N}$ & $\mathrm{~N}$ & $\mathrm{~N}$ & $\mathrm{~N}$ & $\mathrm{~N}$ & $\mathrm{~N}$ & $\mathrm{~N}$ & $\mathrm{~N}$ & $\mathrm{~N}$ & $N$ \\
\hline $2^{\text {nd }}$ & $\mathrm{N}$ & $\mathrm{N}$ & $\mathrm{N}$ & $\mathrm{N}$ & $\mathrm{N}$ & $\mathrm{N}$ & $\mathrm{N}$ & $\mathrm{N}$ & $\mathrm{N}$ & $\mathrm{N}$ & $\mathrm{N}$ & $\mathrm{N}$ & $\mathrm{N}$ & $\mathrm{N}$ & $\mathrm{N}$ & $\mathrm{N}$ & $\mathrm{N}$ & $\mathrm{N}$ &,$+ 20 \%$ \\
\hline $3^{\text {rd }}$ & $\mathrm{N}$ & $\mathrm{N}$ & $\mathrm{N}$ & $\mathrm{N}$ & $\mathrm{N}$ & $\mathrm{N}$ & $\mathrm{N}$ & $\mathrm{N}$ & $\mathrm{N}$ & $\mathrm{N}$ & $\mathrm{N}$ & $\mathrm{N}$ & $\mathrm{N}$ &,$- 10 \%$ & $\mathrm{~N}$ & $N$ & $\mathrm{~N}$ &,$++ 30 \%$ &,$++ 30 \%$ \\
\hline $4^{\text {th }}$ & $\mathrm{N}$ & $\mathrm{N}$ & $\mathrm{N}$ & $\mathrm{N}$ & $\mathrm{N}$ & $\mathrm{N}$ & $\mathrm{N}$ & $\mathrm{N}$ & $\mathrm{N}$ & $\mathrm{N}$ & $\mathrm{N}$ & $\mathrm{N}$ & $\mathrm{N}$ &,$+ 20 \%$ & $\mathrm{~N}$ & $\mathrm{~N}$ & $\mathrm{~N}$ &,$++ 30 \%$ &,$++ 30 \%$ \\
\hline $5^{\text {th }}$ & $\mathrm{N}$ & $\mathrm{N}$ & $\mathrm{N}$ & $\mathrm{N}$ & $\mathrm{N}$ & $\mathrm{N}$ & $\mathrm{N}$ & $\mathrm{N}$ & $\mathrm{N}$ &,$- 10 \%$ & $\mathrm{~N}$ & $\mathrm{~N}$ & $\mathrm{~N}$ &,$+ 20 \%$ & $\mathrm{~N}$ & $\mathrm{~N}$ & $\mathrm{~N}$ &,$+ 20 \%$ &,$++ 30 \%$ \\
\hline $6^{\text {th }}$ & $\mathrm{N}$ & $\mathrm{N}$ & $\mathrm{N}$ & $\mathrm{N}$ & $\mathrm{N}$ & $\mathrm{N}$ & $\mathrm{N}$ & $N$ & $\mathrm{~N}$ &,$- 10 \%$ & $\mathrm{~N}$ & $N$ & $\mathrm{~N}$ & $\mathrm{~N}$ &,$- 10 \%$ & $\mathrm{~N}$ & $N$ &,$+ 20 \%$ &,$++ 30 \%$ \\
\hline $7^{\text {th }}$ & $\mathrm{N}$ & $\mathrm{N}$ & $\mathrm{N}$ & $\mathrm{N}$ & $\mathrm{N}$ & $\mathrm{N}$ & $\mathrm{N}$ & $\mathrm{N}$ & $\mathrm{N}$ &,$- 10 \%$ & $\mathrm{~N}$ & $\mathrm{~N}$ &,$+ 20 \%$ & $\mathrm{~N}$ &,$++ 30 \%$ & $\mathrm{~N}$ & $\mathrm{~N}$ &,$+ 20 \%$ &,$+++ 40 \%$ \\
\hline $8^{\text {th }}$ & $\mathrm{N}$ & $\mathrm{N}$ & $\mathrm{N}$ & $\mathrm{N}$ &,$- 10 \%$ & $\mathrm{~N}$ & $\mathrm{~N}$ & $\mathrm{~N}$ & $\mathrm{~N}$ &,$- 10 \%$ &,$- 10 \%$ & $\mathrm{~N}$ & $\mathrm{~N}$ & $\mathrm{~N}$ &,$++ 30 \%$ & $\mathrm{~N}$ & $\mathrm{~N}$ &,$++ 30 \%$ &,$+++ 40 \%$ \\
\hline $9^{\text {th }}$ & $N$ & $\mathrm{~N}$ & $\mathrm{~N}$ & $\mathrm{~N}$ &,$- 10 \%$ & $\mathrm{~N}$ & $N$ & $N$ & $\mathrm{~N}$ & $\mathrm{~N}$ & $\mathrm{~N}$ & $\mathrm{~N}$ &,$+ 20 \%$ & $\mathrm{~N}$ &,$++ 30 \%$ & $\mathrm{~N}$ & $N$ &,$++ 30 \%$ &,$+++ 40 \%$ \\
\hline $10^{\text {th }}$ & $\mathrm{N}$ & $\mathrm{N}$ & $\mathrm{N}$ & $\mathrm{N}$ & $\mathrm{N}$ & $\mathrm{N}$ & $\mathrm{N}$ & $\mathrm{N}$ & $\mathrm{N}$ & $\mathrm{N}$ &,$+ 20 \%$ & $\mathrm{~N}$ & $\mathrm{~N}$ &,$+ 20 \%$ &,$++ 30 \%$ & $\mathrm{~N}$ & $\mathrm{~N}$ &,$++ 30 \%$ &,$+++ 40 \%$ \\
\hline $11^{\text {th }}$ & $\mathrm{N}$ & $\mathrm{N}$ & $\mathrm{N}$ & $\mathrm{N}$ & $\mathrm{N}$ & $\mathrm{N}$ & $N$ & $N$ &,$- 10 \%$ &,$- 10 \%$ & $\mathrm{~N}$ & $\mathrm{~N}$ &,$+ 20 \%$ &,$+ 20 \%$ &,$++ 30 \%$ & $\mathrm{~N}$ & $\mathrm{~N}$ &,$++ 30 \%$ &,$+++ 40 \%$ \\
\hline $12^{\text {th }}$ & $\mathrm{N}$ & $\mathrm{N}$ & $\mathrm{N}$ & $\mathrm{N}$ & $-10 \%$ & $\mathrm{~N}$ & $\mathrm{~N}$ & $\mathrm{~N}$ & $\mathrm{~N}$ &,$- 10 \%$ & $\mathrm{~N}$ & $\mathrm{~N}$ &,$+ 20 \%$ &,$+ 20 \%$ &,$++ 30 \%$ & $\mathrm{~N}$ & $N$ &,$++ 30 \%$ &,$+++ 40 \%$ \\
\hline $13^{\text {th }}$ & $\mathrm{N}$ & $\mathrm{N}$ & $\mathrm{N}$ & $\mathrm{N}$ &,$- 10 \%$ & $\mathrm{~N}$ & $\mathrm{~N}$ & $\mathrm{~N}$ & $\mathrm{~N}$ & $\mathrm{~N}$ & $\mathrm{~N}$ & $\mathrm{~N}$ & $\mathrm{~N}$ &,$+ 20 \%$ &,$++ 30 \%$ &,$++ 30 \%$ &,$++ 30 \%$ &,$++ 30 \%$ &,++ 40 \\
\hline $14^{\text {th }}$ & $\mathrm{N}$ & $\mathrm{N}$ & $\mathrm{N}$ & $\mathrm{N}$ & $-10 \%$ & $\mathrm{~N}$ & $\mathrm{~N}$ & $\mathrm{~N}$ & $\mathrm{~N}$ &,$- 10 \%$ & $\mathrm{~N}$ & $\mathrm{~N}$ & $\mathrm{~N}$ &,$+ 20 \%$ &,$++ 30 \%$ &,$++ 30 \%$ &,$++ 30 \%$ &,$++ 30 \%$ &,$++ 30 \%$ \\
\hline
\end{tabular}

Table 3: Observation of Piloerection FROM 0-6 h in rats fed with different diets containing different doses of heroin with $\beta$-carotene or without $\beta$-carotene. (Abbreviation: $\mathrm{N}$ no piloerection, -- Doubtful piloerection , + Moderate piloerection ,++ Highst Piloerection more intense, +++ Highest intensity of pilorection, CD=Control diet). The intensity is expressed in $\%$ from the number of animal affected.

\begin{tabular}{|c|c|c|c|c|c|c|c|c|c|c|c|c|c|c|c|c|c|c|c|}
\hline \multirow[t]{2}{*}{$\begin{array}{l}\text { Day of } \\
\text { Heroin } \\
\text { Injection }\end{array}$} & \multirow{2}{*}{\begin{tabular}{|c|} 
O \\
$0-6 \mathrm{~h}$
\end{tabular}} & \multirow{2}{*}{ 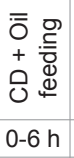 } & \multirow{2}{*}{$\begin{array}{l}1 \\
d \\
0 \\
+ \\
0 \\
0 \\
0 \\
0 \\
0 \\
0 \\
0\end{array}$} & \multicolumn{4}{|c|}{$\begin{array}{c}C D+\beta-\text { Carotene Heroin } \\
\left(\mathrm{LD}_{25}\right)\end{array}$} & \multicolumn{4}{|c|}{$C D+\beta-$ Carotene-Heroin $\left(\mathrm{LD}_{50}\right)$} & \multicolumn{4}{|c|}{$C D+$ Heroin $\left(L^{25}\right)$} & \multicolumn{4}{|c|}{$C D+$ Heroin $\left(L_{50}\right)$} \\
\hline & & & & $0-1 \mathrm{~h}$ & $1-2 \mathrm{~h}$ & $3-4 \mathrm{~h}$ & $4-6 \mathrm{~h}$ & $0-1 \mathrm{~h}$ & $0-2 \mathrm{~h}$ & $3-4 \mathrm{~h}$ & $4-6 \mathrm{~h}$ & $0-1 \mathrm{~h}$ & $0-2 \mathrm{~h}$ & $3-4 \mathrm{~h}$ & $4-6 \mathrm{~h}$ & $0-1 \mathrm{~h}$ & $0-2 \mathrm{~h}$ & $3-4 \mathrm{~h}$ & $4-6 \mathrm{~h}$ \\
\hline $1^{\text {st }}$ & $\mathrm{N}$ & $\mathrm{N}$ & $\mathrm{N}$ & $\mathrm{N}$ & $\mathrm{N}$ & $\mathrm{N}$ & $\mathrm{N}$ & $\mathrm{N}$ & $\mathrm{N}$ & $\mathrm{N}$ & $\mathrm{N}$ & $\mathrm{N}$ & $\mathrm{N}$ & $\mathrm{N}$ & $\mathrm{N}$ &,$+ 20 \%$ & $\mathrm{~N}$ & $\mathrm{~N}$ & $\mathrm{~N}$ \\
\hline $2^{\text {nd }}$ & $\mathrm{N}$ & $\mathrm{N}$ & $\mathrm{N}$ & $\mathrm{N}$ & $\mathrm{N}$ & $\mathrm{N}$ & $\mathrm{N}$ & $\mathrm{N}$ & $\mathrm{N}$ & $\mathrm{N}$ & $\mathrm{N}$ & $\mathrm{N}$ & $\mathrm{N}$ &,$+ 20 \%$ &,$+ 20 \%$ &,$+ 20 \%$ &,$++ 30 \%$ &,$++ 30 \%$ & ++ \\
\hline $3^{\text {rd }}$ & $\mathrm{N}$ & $\mathrm{N}$ & $\mathrm{N}$ & $\mathrm{N}$ & $N$ & $N$ & $\mathrm{~N}$ & $N$ & $N$ & $10 \%$ & $\mathrm{~N}$ & $N$ & 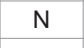 &,$++ 30 \%$ & $\mathrm{~N}$ &,$++ 30 \%$ &,$++ 30 \%$ &,$+ 30 \%$ &,$+ 30 \%$ \\
\hline $4^{\text {th }}$ & $\mathrm{N}$ & $\mathrm{N}$ & $\mathrm{N}$ & $\mathrm{N}$ & $\mathrm{N}$ & $\mathrm{N}$ & $\mathrm{N}$ & $\mathrm{N}$ &,$+ 20 \%$ &,$- 10 \%$ & $\mathrm{~N}$ & $\mathrm{~N}$ & $N$ &, $30 \%$ & $20 \%$ &,$+ 30 \%$ & $\begin{array}{l}+++ \\
, 40 \%\end{array}$ & $40 \%$ &, $30 \%$ \\
\hline $5^{\text {th }}$ & $\mathrm{N}$ & $\mathrm{N}$ & $\mathrm{N}$ & $\mathrm{N}$ &,$- 10 \%$ & $\mathrm{~N}$ & $\mathrm{~N}$ & $\mathrm{~N}$ & $\mathrm{~N}$ & $\mathrm{~N}$ &,$- 10 \%$ & $\mathrm{~N}$ & $\mathrm{~N}$ &,$++ 30 \%$ &,$+ 20 \%$ &,$++ 30 \%$ &,$++ 30 \%$ &,$+++ 40 \%$ &,$+ 30 \%$ \\
\hline $6^{\text {th }}$ & $\mathrm{N}$ & $\mathrm{N}$ & $\mathrm{N}$ & $\mathrm{N}$ & $N$ & $N$ & $\mathrm{~N}$ & $N$ & $\mathrm{~N}$ & ,20\% & $\mathrm{N}$ & IN & $\mathrm{N}$ &,$- 10 \%$ &,$++ 30 \%$ &,$+ 20 \%$ &,$++ 30 \%$ &,$+++ 40 \%$ &,$++ 30 \%$ \\
\hline $7^{\text {th }}$ & $\mathrm{N}$ & $\mathrm{N}$ & $\mathrm{N}$ & $\mathrm{N}$ &,$- 10 \%$ & 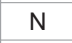 & $\mathrm{N}$ & 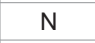 &,$+ 20 \%$ &,$+ 20 \%$ & 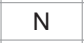 & $\mathrm{N}$ & $\mathrm{N}$ &,$+ 20 \%$ &,$++ 30 \%$ &,$++ 30 \%$ &,$+++ 40 \%$ &,$+++ 40 \%$ &,+ 20 \\
\hline $8^{\text {th }}$ & $\mathrm{N}$ & $\mathrm{N}$ & $\mathrm{N}$ & $\mathrm{N}$ & $\mathrm{N}$ & $\mathrm{N}$ & $\mathrm{N}$ & $\mathrm{N}$ & $N$ & $\mathrm{~N}$ &,$- 10 \%$ & $\mathrm{~N}$ & $\mathrm{~N}$ &,$+ 20 \%$ &,$+ 20 \%$ &,$++ 30 \%$ &,$++ 30 \%$ &,$+++ 40 \%$ &,$+ 20 \%$ \\
\hline $9^{\text {th }}$ & $\mathrm{N}$ & $\mathrm{N}$ & $\mathrm{N}$ & $\mathrm{N}$ &,$- 10 \%$ & $\mathrm{~N}$ & $\mathrm{~N}$ & $\mathrm{~N}$ & $N$ &,$+ 20 \%$ & $\mathrm{~N}$ & $\mathrm{~N}$ & N &,$+ 20 \%$ &,$- 10 \%$ &,$++ 30 \%$ &,$++ 30 \%$ &,$+++ 40 \%$ &,$+++ 40 \%$ \\
\hline $10^{\text {th }}$ & $\mathrm{N}$ & $\mathrm{N}$ & $\mathrm{N}$ & $\mathrm{N}$ & $\mathrm{N}$ & $\mathrm{N}$ & $\mathrm{N}$ & $\mathrm{N}$ & $\mathrm{N}$ &,$+ 20 \%$ & N & $\mathrm{N}$ & $\mathrm{N}$ & $\mathrm{N}$ &,$+ 20 \%$ &,$++ 30 \%$ &,$+++ 40 \%$ &,$+++ 40 \%$ &,$+++ 40 \%$ \\
\hline $11^{\text {th }}$ & $N$ & $\mathrm{~N}$ & $\mathrm{~N}$ & $\mathrm{~N}$ & $\mathrm{~N}$ & $\mathrm{~N}$ & $\mathrm{~N}$ & $\mathrm{~N}$ & $\mathrm{~N}$ & $\mathrm{~N}$ & $\mathrm{~N}$ & $\mathrm{~N}$ & $N$ & $\mathrm{~N}$ &,$+ 20 \%$ &,$+ 30 \%$ &,$+++ 40 \%$ &,$++ 30 \%$ &,$+++ 40 \%$ \\
\hline $12^{\text {th }}$ & $N$ & $\mathrm{~N}$ & $\mathrm{~N}$ & s & $-10 \%$ & $\mathrm{~N}$ & & & & $\mathrm{~N}$ & & 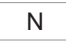 & & & $\%$ & $N$ &,$+++ 40 \%$ &,$+++ 40 \%$ &,$+++ 40 \%$ \\
\hline $13^{\text {th }}$ & $N$ & $\mathrm{~N}$ & $\mathrm{~N}$ & $\mathrm{~N}$ & $-10 \%$ & $\mathrm{~N}$ &,$- 10 \%$ &,$- 10 \%$ & 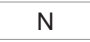 &,$+ 20 \%$ &,$+ 20 \%$ & TV &,$+ 20 \%$ &,$+ 20 \%$ &,$+ 20 \%$ &,$++ 30 \%$ &,$+++ 40 \%$ &,$++ 30 \%$ &,$++ 30 \%$ \\
\hline $14^{\text {th }}$ & $\mathrm{N}$ & $\mathrm{N}$ & $\mathrm{N}$ & $\mathrm{N}$ & $-10 \%$ & $\mathrm{~N}$ &,$- 10 \%$ &,$- 10 \%$ & $\mathrm{~N}$ &,$+ 20 \%$ &,$+ 20 \%$ & $\mathrm{~N}$ &,$+ 20 \%$ &,$+ 20 \%$ &,$+ 20 \%$ &,$++ 30 \%$ &,$++ 30 \%$ &,$+++ 40 \%$ &,$+++ 40 \%$ \\
\hline
\end{tabular}

Table 4: Etching Behavior observed within 4-6 $\mathrm{h}$ in rats fed with different diets containing different doses of heroin with $\beta$-carotene or without $\beta$-carotene.

Abbreviation: $\mathrm{N}=$ No etching, - A few cases of doubtful etching ,+ Frequent etching, ++ Higher No. of etchings, +++ Highest cases of etchings; CD=Control diet) The intensity is expressed in $\%$ from the number of animal affected. 


\begin{tabular}{|c|c|c|c|c|c|c|c|c|c|c|c|c|c|c|c|c|c|c|c|}
\hline \multirow[t]{2}{*}{$\begin{array}{l}\text { Day of } \\
\text { Heroin } \\
\text { Injection }\end{array}$} & \multirow{2}{*}{\begin{tabular}{|c|} 
Oे \\
$0-4 h$
\end{tabular}} & \multirow{2}{*}{ 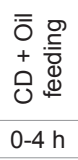 } & \multirow{2}{*}{ 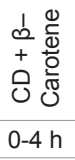 } & \multicolumn{4}{|c|}{$\begin{array}{c}\mathrm{CD}+\beta-\text { Carotene Heroin } \\
\left(\mathrm{LD}_{25}\right)\end{array}$} & \multicolumn{4}{|c|}{$\begin{array}{c}\mathrm{CD}+\beta-\text { Carotene-Heroin } \\
\left(\mathrm{LD}_{50}\right)\end{array}$} & \multicolumn{4}{|c|}{$C D+$ Heroin $\left(L_{25}\right)$} & \multicolumn{4}{|c|}{$C D+$ Heroin $\left(L_{50}\right)$} \\
\hline & & & & $0-1 \mathrm{~h}$ & $1-2 \mathrm{~h}$ & $2-3 h$ & $3-4 \mathrm{~h}$ & $0-1 \mathrm{~h}$ & $1-2 \mathrm{~h}$ & $2-3 \mathrm{~h}$ & $3-4 h$ & $0-1 \mathrm{~h}$ & $1-2 \mathrm{~h}$ & $2-3 \mathrm{~h}$ & $3-4 \mathrm{~h}$ & $0-1 \mathrm{~h}$ & $1-2 \mathrm{~h}$ & $2-3 \mathrm{~h}$ & 3-4 h \\
\hline $1^{\text {st }}$ & $\mathrm{N}$ & $\mathrm{N}$ & $\mathrm{N}$ & $\mathrm{N}$ & $\mathrm{N}$ & $\mathrm{N}$ & $\mathrm{N}$ & $\mathrm{N}$ & $\mathrm{N}$ & $\mathrm{N}$ & $\mathrm{N}$ & $\mathrm{N}$ & $\mathrm{N}$ & $\mathrm{N}$ & $\mathrm{N}$ & $\mathrm{N}$ & $\mathrm{N}$ & $\mathrm{N}$ & $\mathrm{N}$ \\
\hline $2^{\text {nd }}$ & $\mathrm{N}$ & $\mathrm{N}$ & $\mathrm{N}$ & $\mathrm{N}$ & $\mathrm{N}$ & $\mathrm{N}$ & $\mathrm{N}$ & $\mathrm{N}$ & $\mathrm{N}$ & $\mathrm{N}$ & $\mathrm{N}$ & $\mathrm{N}$ & $\mathrm{N}$ & $\mathrm{N}$ & $\mathrm{N}$ & $\mathrm{N}$ & $\mathrm{N}$ &,$++ 30 \%$ &,$++ 30 \%$ \\
\hline 3 rs & $\mathrm{N}$ & $\mathrm{N}$ & $\mathrm{N}$ & $\mathrm{N}$ & $\mathrm{N}$ & $\mathrm{N}$ & $\mathrm{N}$ & $\mathrm{N}$ &,$+ 20 \%$ & $\mathrm{~N}$ & $\mathrm{~N}$ & $\mathrm{~N}$ & $\mathrm{~N}$ & $\mathrm{~N}$ &,$+ 20 \%$ & $\mathrm{~N}$ & $\mathrm{~N}$ &,$++ 30 \%$ &,$++ 30 \%$ \\
\hline $4^{\text {th }}$ & $\mathrm{N}$ & $\mathrm{N}$ & $\mathrm{N}$ & $\mathrm{N}$ & $N$ & $\mathrm{~N}$ & $\mathrm{~N}$ & $N$ &,$+ 20 \%$ & $\mathrm{~N}$ & $\mathrm{~N}$ & $N$ & $N$ & $\mathrm{~N}$ &,$+ 20 \%$ & $\mathrm{~N}$ & $\mathrm{~N}$ &,$+ 30 \%$ &,$++ 30 \%$ \\
\hline $5^{\text {th }}$ & $\mathrm{N}$ & $\mathrm{N}$ & $\mathrm{N}$ & $\mathrm{N}$ & $N$ & $\mathrm{~N}$ & $N$ & $\mathrm{~N}$ & $\mathrm{~N}$ & $\mathrm{~N}$ & $N$ & $\mathrm{~N}$ & $\mathrm{~N}$ &,$+ 20 \%$ & $N$ &,$+ 20 \%$ & $\mathrm{~N}$ &,$+ 30 \%$ &,$+++ 40 \%$ \\
\hline $6^{\text {th }}$ & $\mathrm{N}$ & $\mathrm{N}$ & $\mathrm{N}$ & $\mathrm{N}$ & $N$ & $N$ & $N$ & $N$ & $\mathrm{~N}$ & $\mathrm{~N}$ & $\mathrm{~N}$ & $\mathrm{~N}$ & $\mathrm{~N}$ &,$++ 30 \%$ & $N$ &,$+ 20 \%$ & $\mathrm{~N}$ &,$++ 30 \%$ &,$+++ 40 \%$ \\
\hline $7^{\text {th }}$ & $\mathrm{N}$ & $\mathrm{N}$ & $\mathrm{N}$ & $\mathrm{N}$ & $\mathrm{N}$ & $\mathrm{N}$ & $\mathrm{N}$ & $\mathrm{N}$ & $\mathrm{N}$ & $\mathrm{N}$ & $\mathrm{N}$ & $\mathrm{N}$ & $\mathrm{N}$ &,$++ 30 \%$ &,$+ 20 \%$ &,$+ 20 \%$ & $\mathrm{~N}$ &,$++ 30 \%$ &,$+++ 40 \%$ \\
\hline $8^{\text {th }}$ & $\mathrm{N}$ & $\mathrm{N}$ & $\mathrm{N}$ & $\mathrm{N}$ & $N$ &,$+ 20 \%$ & $\mathrm{~N}$ & $\mathrm{~N}$ &,$+ 20 \%$ &,$+ 20 \%$ & $\mathrm{~N}$ & $\mathrm{~N}$ & $\mathrm{~N}$ &,$++ 30 \%$ &,$+ 20 \%$ & $\mathrm{~N}$ & $\mathrm{~N}$ &,$++ 30 \%$ &,$+++ 40 \%$ \\
\hline $9^{\text {th }}$ & $\mathrm{N}$ & $\mathrm{N}$ & $\mathrm{N}$ & $\mathrm{N}$ & $\mathrm{N}$ & $\mathrm{N}$ & $\mathrm{N}$ & $\mathrm{N}$ & $\mathrm{N}$ & $\mathrm{N}$ & $\mathrm{N}$ & $\mathrm{N}$ & $\mathrm{N}$ &,$++ 30 \%$ & $N$ & $\mathrm{~N}$ & $\mathrm{~N}$ &,$++ 30 \%$ &,$+++ 40 \%$ \\
\hline $10^{\text {th }}$ & $\mathrm{N}$ & $\mathrm{N}$ & $\mathrm{N}$ & $\mathrm{N}$ & $\mathbf{N}$ & $\mathrm{N}$ & $\mathrm{N}$ & $\mathrm{N}$ & $\mathrm{N}$ & $\mathrm{N}$ & $\mathrm{N}$ & $\mathrm{N}$ & $\mathrm{N}$ &,$++ 30 \%$ & $\mathrm{~N}$ & $N$ & $\mathrm{~N}$ &,$++ 30 \%$ &,$+++ 40 \%$ \\
\hline $11^{\text {th }}$ & $\mathrm{N}$ & $\mathrm{N}$ & $\mathrm{N}$ & $\mathrm{N}$ & $N$ & $N$ & $\mathrm{~N}$ & $\mathrm{~N}$ & $\mathrm{~N}$ & $\mathrm{~N}$ & $\mathrm{~N}$ & $\mathrm{~N}$ & $\mathrm{~N}$ &,$++ 30 \%$ & $\mathrm{~N}$ & $N$ & $\mathrm{~N}$ &,$++ 30 \%$ &,$+++ 40 \%$ \\
\hline $12^{\text {th }}$ & $\mathrm{N}$ & $\mathrm{N}$ & $\mathrm{N}$ & $\mathrm{N}$ & $N$ & $\mathrm{~N}$ & $N$ & $N$ & $\mathrm{~N}$ & $N$ & $\mathrm{~N}$ & $\mathrm{~N}$ & $\mathrm{~N}$ &,$++ 30 \%$ & $\mathrm{~N}$ & $\mathrm{~N}$ & $\mathrm{~N}$ &,$+++ 40 \%$ &,$++++ 50 \%$ \\
\hline 13th & $\mathrm{N}$ & $\mathrm{N}$ & $\mathrm{N}$ & $\mathrm{N}$ & $\mathrm{N}$ &,$+ 20 \%$ &,$- 10 \%$ & $\mathrm{~N}$ & $\mathrm{~N}$ & $\mathrm{~N}$ &,$+ 20 \%$ & $\mathrm{~N}$ &,$- 10 \%$ &,$++ 30 \%$ & $\mathrm{~N}$ & $N$ &,$+ 20 \%$ &,$++ 30 \%$ &,+++ 40 \\
\hline 14th & $\mathrm{N}$ & $\mathrm{N}$ & $\mathrm{N}$ & $\mathrm{N}$ & $N$ &,$+ 20 \%$ &,$-- 10 \%$ & $\mathrm{~N}$ & $N$ & $\mathrm{~N}$ &,$+ 20 \%$ & $N$ &,$- 10 \%$ &,$++ 30 \%$ & $N$ & $\mathrm{~N}$ &,$+ 20 \%$ &,$++ 30 \%$ &,+++ 40 \\
\hline
\end{tabular}

Table 5: Jumping behavious: within 0-6 h in rats fed with different diets containing different doses of heroin with $\beta$-carotene or without $\beta$-carotene. (Abbreviation: $\mathrm{N}=\mathrm{N}$

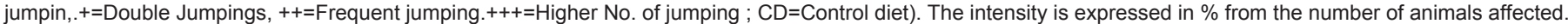

\begin{tabular}{|c|c|c|c|c|c|c|c|c|c|c|c|c|c|c|c|c|c|c|c|}
\hline \multirow[t]{2}{*}{$\begin{array}{l}\text { Day of } \\
\text { Heroin } \\
\text { Injection }\end{array}$} & \multirow{2}{*}{\begin{tabular}{|c|}
0 \\
$0-4 h$ \\
\end{tabular}} & \multirow{2}{*}{ 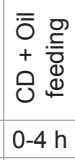 } & \multirow{2}{*}{ 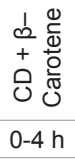 } & \multicolumn{4}{|c|}{$C D+\beta-$ Carotene Heroin $\left(\mathrm{LD}_{25}\right)$} & \multicolumn{4}{|c|}{$\begin{array}{c}C D+\beta-\text { Carotene-Heroin } \\
\left(\mathrm{LD}_{50}\right)\end{array}$} & \multicolumn{4}{|c|}{$C D+$ Heroin $\left(\mathrm{LD}_{25}\right)$} & \multicolumn{4}{|c|}{$C D+$ Heroin $\left(L_{50}\right)$} \\
\hline & & & & $0-1 \mathrm{~h}$ & $1-2 \mathrm{~h}$ & $2-3 \mathrm{~h}$ & $4-6 \mathrm{~h}$ & $0-1 \mathrm{~h}$ & $1-2 \mathrm{~h}$ & $2-3 \mathrm{~h}$ & $4-6 \mathrm{~h}$ & $0-1 \mathrm{~h}$ & $1-2 \mathrm{~h}$ & $2-3 \mathrm{~h}$ & $4-6 \mathrm{~h}$ & $0-1 \mathrm{~h}$ & $1-2 \mathrm{~h}$ & $2-3 \mathrm{~h}$ & $4-6 \mathrm{~h}$ \\
\hline $1^{\text {st }}$ & $\mathrm{N}$ & $\mathrm{N}$ & $\mathrm{N}$ & $\mathrm{N}$ & $\mathrm{N}$ & $\mathrm{N}$ & $\mathrm{N}$ & $\mathrm{N}$ & $\mathrm{N}$ & $\mathrm{N}$ & $\mathrm{N}$ & $\mathrm{N}$ & $\mathrm{N}$ & $\mathrm{N}$ & $\mathrm{N}$ & $\mathrm{N}$ & $\mathrm{N}$ & $\mathrm{N}$ & $\mathrm{N}$ \\
\hline $2^{\text {nd }}$ & $\mathrm{N}$ & $\mathrm{N}$ & $\mathrm{N}$ & $\mathrm{N}$ & $\mathrm{N}$ & $\mathrm{N}$ & NN & $\mathrm{N}$ & $\mathrm{N}$ & $\mathrm{N}$ & $\mathrm{N}$ & $\mathrm{N}$ & $\mathrm{N}$ & $\mathrm{N}$ & $\mathrm{N}$ & $\mathrm{N}$ & $\mathrm{N}$ &,$+ 20 \%$ &,$+ 20 \%$ \\
\hline $3^{\text {rd }}$ & $\mathrm{N}$ & $\mathrm{N}$ & $\mathrm{N}$ & $\mathrm{N}$ & $\mathrm{N}$ & $\mathrm{N}$ & $\mathrm{N}$ & $\mathrm{N}$ & $\mathrm{N}$ & $\mathrm{N}$ & $\mathrm{N}$ & $\mathrm{N}$ & $\mathrm{N}$ & $\mathrm{N}$ & $\mathrm{N}$ & $\mathrm{N}$ & $\mathrm{N}$ &,$+ 20 \%$ &,$++ 30 \%$ \\
\hline $4^{\text {th }}$ & $\mathrm{N}$ & $\mathrm{N}$ & $\mathrm{N}$ & $\mathrm{N}$ & $\mathrm{N}$ & $\mathrm{N}$ & $\mathrm{N}$ & $\mathrm{N}$ & $\mathrm{N}$ & $\mathrm{N}$ & +4 & $\mathrm{~N}$ & $\mathrm{~N}$ & $\mathrm{~N}$ & +5 & $\mathrm{~N}$ &,$+ 20 \%$ &,$++ 30 \%$ &,$++ 30 \%$ \\
\hline $5^{\text {th }}$ & $\mathrm{N}$ & $\mathrm{N}$ & $\mathrm{N}$ & $\mathrm{N}$ & $\mathrm{N}$ & $\mathrm{N}$ & $\mathrm{N}$ & $\mathrm{N}$ & $\mathrm{N}$ &,$+ 20 \%$ & $\mathrm{~N}$ & $\mathrm{~N}$ & $\mathrm{~N}$ &,$+ 20 \%$ &,$+ 20 \%$ & $\mathrm{~N}$ & $\mathrm{~N}$ &,$+ 20 \%$ &,$+++ 40 \%$ \\
\hline $6^{\text {th }}$ & $\mathrm{N}$ & $\mathrm{N}$ & $\mathrm{N}$ & $\mathrm{N}$ & $\mathrm{N}$ & $\mathrm{N}$ & $\mathrm{N}$ & $\mathrm{N}$ & $\mathrm{N}$ &,$+ 20 \%$ & $\mathrm{~N}$ & $\mathrm{~N}$ & $\mathrm{~N}$ &,$+ 20 \%$ & $\mathrm{~N}$ & $\mathrm{~N}$ & $\mathrm{~N}$ &,$+++ 40 \%$ &,$++ 30 \%$ \\
\hline $7^{\text {th }}$ & $\mathrm{N}$ & $\mathrm{N}$ & $\mathrm{N}$ & $\mathrm{N}$ & $\mathrm{N}$ & $\mathrm{N}$ & $\mathrm{N}$ & $\mathrm{N}$ & $\mathrm{N}$ & $\mathrm{N}$ &,$+ 20 \%$ & $\mathrm{~N}$ & $\mathrm{~N}$ &,$+ 20 \%$ & $\mathrm{~N}$ & $\mathrm{~N}$ &,$+ 20 \%$ &,$++ 30 \%$ &,$++ 30 \%$ \\
\hline $8^{\text {th }}$ & $\mathrm{N}$ & $\mathrm{N}$ & $\mathrm{N}$ & $\mathrm{N}$ & $\mathrm{N}$ & $\mathrm{N}$ & 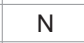 & $\mathrm{N}$ & $\mathrm{N}$ & $\mathrm{N}$ &,$+ 20 \%$ & $\mathrm{~N}$ & $-20 \%$ & 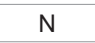 & $N$ & $\mathrm{~N}$ & $\mathrm{~N}$ &,$++ 30 \%$ &,$++ 30 \%$ \\
\hline $9^{\text {th }}$ & $\mathrm{N}$ & $\mathrm{N}$ & $\mathrm{N}$ & $\mathrm{N}$ & $\mathrm{N}$ & $\mathrm{N}$ & $\mathrm{N}$ & $\mathrm{N}$ & $\mathrm{N}$ &,$+ 20 \%$ &,$+ 20 \%$ & $\mathrm{~N}$ &,$+ 20 \%$ &,$+ 20 \%$ &,$+ 20 \%$ & $\mathrm{~N}$ & $\mathrm{~N}$ &,$++ 30 \%$ &,$++ 30 \%$ \\
\hline $10^{\text {th }}$ & $\mathrm{N}$ & $\mathrm{N}$ & $\mathrm{N}$ & $\mathrm{N}$ & $\mathrm{N}$ & $\mathrm{N}$ & $\mathrm{N}$ & $\mathrm{N}$ & $\mathrm{N}$ &,$+ 20 \%$ & $\mathrm{~N}$ & $\mathrm{~N}$ & $\mathrm{~N}$ &,$+ 20 \%$ &,$+ 20 \%$ & $\mathrm{~N}$ & $\mathrm{~N}$ &,$++ 30 \%$ &,$++ 30 \%$ \\
\hline $11^{\text {th }}$ & $\mathrm{N}$ & $\mathrm{N}$ & $\mathrm{N}$ & $\mathrm{N}$ & $\mathrm{N}$ & $\mathrm{N}$ & $\mathrm{N}$ & $\mathrm{N}$ & $\mathrm{N}$ & $\mathrm{N}$ & $\mathrm{N}$ & $\mathrm{N}$ & $\mathrm{N}$ &,$+ 20 \%$ &,$+ 20 \%$ & $\mathrm{~N}$ & $\mathrm{~N}$ &,$+++ 40 \%$ &,$++ 30 \%$ \\
\hline $12^{\text {th }}$ & $\mathrm{N}$ & $\mathrm{N}$ & $\mathrm{N}$ & $\mathrm{N}$ & $\mathrm{N}$ & $\mathrm{N}$ & $\mathrm{N}$ & $\mathrm{N}$ & $\mathrm{N}$ & $\mathrm{N}$ & $\mathrm{N}$ & $\mathrm{N}$ & $\mathrm{N}$ &,$+ 20 \%$ &,$+ 20 \%$ & $\mathrm{~N}$ & $\mathrm{~N}$ &,$+++ 40 \%$ &,$++ 30 \%$ \\
\hline $13^{\text {th }}$ & $\mathrm{N}$ & $\mathrm{N}$ & $\mathrm{N}$ & $\mathrm{N}$ & $\mathrm{N}$ & $\mathrm{N}$ &,$- 10 \%$ & -;10\% & $\mathrm{N}$ &,$+ 20 \%$ &,$+ 20 \%$ & $\mathrm{~N}$ & $\mathrm{~N}$ &,$+ 20 \%$ &,$+++ 40 \%$ & $\mathrm{~N}$ & $\mathrm{~N}$ &,$++ 30 \%$ &,$+++ 40 \%$ \\
\hline $14^{\text {th }}$ & $\mathrm{N}$ & $\mathrm{N}$ & $\mathrm{N}$ & $\mathrm{N}$ & $\mathrm{N}$ & $\mathrm{N}$ &,$- 10 \%$ & $-; 10 \%$ & $\mathrm{~N}$ &,$+ 20 \%$ &,$+ 20 \%$ & $\mathrm{~N}$ & $\mathrm{~N}$ &,$+ 20 \%$ &,$+++ 40 \%$ & $\mathrm{~N}$ & $\mathrm{~N}$ &,$++ 30 \%$ &,$+++ 40 \%$ \\
\hline
\end{tabular}

Table 6: Variation of wet shakes within $4-6 \mathrm{~h}$ in rats fed with different diets containing different doses of heroin with $\beta$-carotene or without $\beta$-carotene. ( Abbreviation: $N=$ No wet shake ; +=Double wet shake; ++=Frequent wet shakes; +++=Highest wet shakes ; CD=Control diet) The intensity is expressed in \% from the number of animal affected.

probably due to nutritional effects of $\beta$-carotene and its function of converting into retinol, as well as its other manifold functions.

The present diet used for feeding the rats was followed after [11], and also from our earlier reports [10]. The diet amount, 10g/100g body weight of the animal showed continuous growth. The suitability and acceptance of the diet has been tested by administrating $8,10,12,15$ g per $100 \mathrm{~g}$ body weight of the animal. It has been found that $10 \mathrm{~g} / 100 \mathrm{~g}$ body weight has been accepted as normal consumption rather than the other amounts. $\beta$-carotene added diets showed greater growth than the control or control + oil as well as the other diets such as $\beta$-carotene + heroin of both $\mathrm{LD}_{25}$ and $\mathrm{LD}_{50}$ doses of heroin showed growth with various withdrawal symptoms. The drug has affected growth as well as triggering various abnormal behaviours of the rats.

The various withdrawal symptoms were noticed after the administration of $\beta$-carotene and heroin added diets of different concentrations. The reduction of water intake, is significantly reduced in the diets added with heroin $\mathrm{LD}_{25}$ and $\mathrm{LD}_{50}$ doses. $\beta$-carotene added diets showed better water intake than the heroin added diets. In (Tables
2-6) the various effects are shown. Several workers have reported the loss of weight in the heroin treated experimental animals [2,9,14,2023] have opined that there may be two possible mechanisms, where there are altered levels of anabolic hormones with decrease of food intake. In the present investigation also, it has been found that there is a decrease of food intake in batches of the animals fed with heroin $\mathrm{LD}_{25}$ and $\mathrm{LD}_{50}$. However $\beta$-carotene + heroin fed animals with varying doses of heroin did not show any significant low intake of food. The support of $\beta$-carotene in maintaining normal growth or increase of body weight is due to its multifaceted nutritional role and its capacity of being able to be converted it into retinol. It is a potent anti-oxidant and supports growth.

The number of jumps is a convenient and reliable index of the withdrawal syndrome in mice [24]. The frequency of jumping and the mode of jumping obtained in the experiment are supported by [15] who described that the number of jumps increases with the increase of the dose of morphine. Similar results were also obtained in heroin treatment significant number. Of jumping were associated with heroin $\left(\mathrm{LD}_{50}\right)$ treatment during 3-6 hrs from $6^{\text {th }}$ to $12^{\text {th }}$ dose. Except in very 
few cases, the $\beta$-carotene + heroin $\left(\mathrm{LD}_{50}\right)$ did not show any jumping. $\beta$-carotene could play a positive role in preventing the withdrawal symptoms such as jumping in the heroin administered rats.

After injecting doses of heroin on alternate days, there was no further increase in the number of jumps after six days. The same results were obtained in morphine injection [24]. The number of jumps correlates with other withdrawal syndromes. These results suggest that in later type of experiment a study has been achieved where withdrawal between doses balances the continued administration of the same dose of morphine [15] and hence heroin also.

Wet shakes (or body shakes) constitute a widely used and reliable criterion for narcotic withdrawal. The type of wet shakes observed was supported by the findings of [14].

The decrease of water intake after heroin treatment has been shown by several workers [25-27]. Naloxane, a narcotic drug suppressant affects the food and water intake. Some of the hypothesis of above findings stated that after each daily heroin injection, the rats demonstrated relatively little intracranial self stimulation on food and water. The several withdrawal symptoms shown in the present experiments are consistent with earlier findings [13-14]. Since $\beta$ - carotene is effective in the therapy of heroin addicts and since $\beta$-carotene has been shown to partially prevent the loss of body weight by heroin, it could not partially block the abrupt withdrawal reaction after heroin addiction. However, it is not clear whether the weight loss can be attributed primarily due to the decrease in food and water consumption during withdrawal or due to the loss of bulk and fluids through increased defecation and urination during withdrawal.

\section{Acknowledgement}

We thank Hoffman La Roch, Basel, Switzerland for providing the standards retinoids. Thanks are due to the care takers of the animal house, Department of Zoology, Gauhati University, Gauhati for providing necessary help during the experiments.

\section{References}

1. Akera T, Broody TM (1968) The addiction cycle to narcotics in the rat and its relation to cathecholamines, Biochem Pharmacol 17: 675-688.

2. Stolerman IP, Johnson CA, Bunker P, Jarvik ME (1975) Weight loss and shock elicited aggression as indices of morphine abstinence in rats. Psychopharmacologia 45: 157- 161

3. Nyswander M (1956) The Drug Addict as a Patient. Grune and Stratton, New York, USA.

4. Martin WR (1972) Pathophysiology of narcotic addiction: Possible roles of protracted abstinence in relapse. In Drug Abuse: Proceedings of the international Conference (CJD Zarafonetis, ed-) Philadelphia: 153- 159.

5. Buckett WR (1964) A new test for morphine- like physical dependence (addiction liability) in rats. Psychopharmacologia 6: 410-416.

6. Gianutsos G, Hynes MD, Puri SK, Drawbaugh RB, Lal H (1974) Effect of apomorphine and nigrostriatal lesions on aggression and striatal dopamine turnover during morphine withdrawal: Evidence for dopaminergic supersensitivity in protected abstinence. Psychopharmacologia 34: 37-44.

7. Wikler A, Green P, Smith H, Pescor FT (1960) Use of benzimidazole derivative with potent morphine- like properties orally as a presumptive reinforce in conditioning of drug seeking behaviour in rats. Fed Proc Fed Amer Soc Exp Boil 19: 22-28.

8. Martin WR, Solan, JW (1971) The pathophysiology of morphine dependence and its treatment with opioid antagonists. Pharmakopsychiatry 14: 260- 270.

9. Martin WR, Wikler A, Eades CG, Pescor FT (1963) Tolerance to and physical dependence on morphine in rats. Psychopharmacology 4: 247-260.

10. Saha PK, Goswami UC, Dutta SK, De AK (2006) $\beta$-Carotene prevents heroin induced changes insome serum enzymes in rats. Sci \& Cult 72: 141-146.
11. Santhanam U, Lalitha VS, Bhide SV (1987) Carcinogenicity of diethylnitrosamine in vitamin A deficient mice. Int J Cancer 40: 784-787.

12. Omkar (1994) Concept of toxicology. SLN Chand \& Co. Jalandhar, India

13. Janseen PA, Niemegeers CJ, Schellkens KH (1965) Is it possible to predict the clinical effects of neuroleptic drugs(major tranquillizers)mfrom animal data? I. "Neuroleptic activity spectra" for rats. Arzneimittalforshung 15: 104-117.

14. Gianutsos G, Martin H, Richard D, Harbanas L (1975) The narcotic withdrawa syndrome in the rat In: Methods in narcotic Research. Modern PharmacologyToxicology. (A series of monographs and Text books) ends. Seymour Ehrenpreis and Amos Neidle. (C) marshall Dekker, Inc. New York.

15. Marshall I (1972). The morphine withdrawal syndrome and its modification by drug. In: Agonist and Antagonist actions of narcotic analegesic drugs. Eds. H W. Kosterlitz H. O. J. Collier, and J. E. Villarreal. (C) British pharmacological Society. Macmillan publishers.

16. Krinsky NI (1993) Micronutrients and their influence on mutagenicity and malignant transformation. Ann NY Acad Sci 686: 229-242.

17. Goswami UC, Sharma N (2005) Efficiency of a few retinoids and carotenoids in vivo in controlling the benzo[a] pyrene-induced forestomach tumor in female Swiss mice. Br J Nutr 94: 540-543.

18. Sharma N, Goswami UC (2011) Functioning of lycopene, a non-provitamin a carotenoid in mammalian system: A review. Proc Zool Soc DOI 10.1007/ S1295-011-0005-0

19. Thomas JA (1995) Drug -nutrient interaction. Nutr Rev 53: 271-282

20. Mucha RF, Kalant H (1979) Increased weight gain as a morphine withdrawal response in rats. Pharmacol Biochem Behav 11: 197- 201.

21. Koga N, George R (1974) Narcotics and the Hypothalamus. Edited by E. Zimmerman and R. George. New York: Raven Press. Pp. 137-157

22. Kumar SP, Joshi HC, Shivnani GA (1971) Feed and water deprivation in chickens. Indian Vet J 48: 545-547.

23. Koga Y, Inukai T (1981) Characterization of withdrawal syndrome of morphine-dependent rats prepared by intermittent infusion technique. Psychopharmacology (Berl) 73: 230- 235.

24. Marshall I, Weinstock M (1971) Quantitative method for assessing one symptom of the withdrawal syndrome in mice after chronice morphine administration. Nature 234: 223- 224.

25. Frank H, Rogers GH (1979) The suppressant effects of naloxone on food and water intake in the rat. Behav Neural Biol 26: 23-40.

26. Sanger DJ, McCarthy PS, Metcalf G (1981) The effect of opiate antagonists on food intake are stereospecific. Neuropharmacology 20: 45- 47.

27. Koob GF, Spector NH, Meyrhoff JL (1975) Effects of heroin on lever pressing fo intracranial self- sitimulation, food and water in the rat. Psychopharmacologia 42: 231- 234. 